\title{
Efeitos do plantio direto e da consorciação soja-milho sobre inimigos naturais e pragas ${ }^{(1)}$
}

\begin{abstract}
Francisco Jorge Cividanes ${ }^{(2)}$ e José Carlos Barbosa ${ }^{(3)}$
Resumo - Procurou-se avaliar os efeitos do plantio direto e da consorciação soja (Glycine max (L.) Merrill) e milho (Zea mays L.) sobre pragas e inimigos naturais. Os tratamentos constituíram um fatorial 3 x 2 (monocultura de soja, monocultura de milho, consorciação soja-milho x plantio direto, plantio convencional), em blocos casualizados. Os insetos foram amostrados pelo método do pano, rede entomológica, procura visual e armadilha de sucção. Entre os insetos-pragas do milho, Maecolaspis assimilis ocorreu em maior número no sistema de plantio convencional; o mesmo ocorreu com os predadores Cycloneda sanguinea e Doru sp. Por outro lado, M. assimilis e o predador Toxomerus sp. foram mais numerosos na monocultura de milho em relação à cultura do milho consorciado com soja. Dos insetos-pragas da soja, destacaram-se pelo maior número Anticarsia gemmatalis e Diabrotica gracilenta, no sistema de plantio convencional, e o mesmo aconteceu com a espécie da família Trichogrammatidae, enquanto as espécies da família Eulophidae foram mais numerosas na soja sob sistema de plantio direto. Na soja consorciada com milho foi maior o número de insetos-pragas Megalotomus sp. e Maecolaspis sp. e dos inimigos naturais Geocoris sp., Lebia concina, Orius sp., Braconidae e Scelionidae.
\end{abstract}

Termos para indexação: Glycine max, Zea mays, insetos, controle de pragas.

\section{Effects of no-tillage and of soybean-corn intercropping on natural enemies and pests}

\begin{abstract}
This study evaluated the effects of no-tillage and of strip intercropping of soybean (Glycine $\max$ (L.) Merrill) and corn (Zea mays L.) on the abundance of pests and natural enemies. The plots were arranged in a randomized complete block design for a $3 \times 2$ factorial experiment (soybean monoculture, corn monoculture, strip intercropping of soybean-corn $\mathrm{x}$ no-tillage and conventional tillage). Insects were sampled by sweep net, ground cloth, visual search and suction net. Among the corn insect pests, Maecolaspis assimilis occurred in highest number in the no-tillage system, the same being observed with the predators Cycloneda sanguinea and Doru sp. On the other hand, M. assimilis and the predator Toxomerus sp. were more abundant in corn monoculture than in corn of the strip-intercropping system. Considering the soybean insect pests, highest number of Anticarsia gemmatalis and Diabrotica gracilenta was found in the conventional tillage system; the same happened to the Trichogrammatidae species, while species of Eulophidae family were more numerous in soybean under no-tillage system. Soybean of the strip-intercropping system showed highest abundance of the insect pests Megalotomus sp. and Maecolaspis sp. and the natural enemies Geocoris sp., Lebia concina, Orius sp., Braconidae and Scelionidae.
\end{abstract}

Index terms: Glycine max, Zea mays, insects, pest control.

(1) Aceito para publicação em 5 de maio de 2000. Financiado pela FAPESP.

(2) Universidade Estadual Paulista (UNESP), Faculdade de Ciências Agrárias e Veterinárias (FCAV), Dep. de Fitossanidade, CEP 14870-000 Jaboticabal, SP. E-mail: fjcivida@fcav.unesp.br

(3) UNESP, FCAV, Dep.de Ciências Exatas, CEP 14870-000 Jaboticabal, SP. E-mail: jbarbosa@fcav.unesp.br

\section{Introdução}

O interesse em uma agricultura com pouco uso de insumos tem aumentado a importância do controle biológico natural de pragas. Estudos têm mostrado a grande abundância de insetos predadores nas culturas da soja e do milho (Hsin et al., 1979; 
Bechinski \& Pedigo, 1981), e existem evidências de que estes inimigos naturais afetam a dinâmica populacional de pragas daquelas culturas (Best \& Beegle, 1977; Fuller, 1988).

Tanto o plantio direto como a consorciação de culturas são fatores de diversificação do agroecossistema, por aumentarem a diversidade estrutural e de espécies, as quais podem afetar a densidade de insetos (Emden \& Williams, 1974). Estudos sobre o efeito da diversidade de agroecossistemas sobre populações de insetos têm mostrado que a consorciação de culturas e o plantio direto podem aumentar ou diminuir a densidade populacional de pragas e de inimigos naturais (Andow, 1991). Em geral, as populações de insetos fitófagos são, freqüentemente, mais baixas em policulturas do que em monoculturas (Risch et al., 1983). Com relação a inimigos naturais, House \& Stinner (1983) observaram que a diversidade de espécies de carabídeos foi significativamente maior em soja de plantio direto do que de plantio convencional. Brust et al. (1986) mostraram que em sistema de plantio direto do milho, predadores foram o principal fator a causar a redução dos danos causados por Agrotis ipsilon, enquanto Letourneau (1987) determinou que a taxa de parasitismo de Trichogramma pretiosum e Chelonus sp. foi maior em culturas consorciadas de milho-Cucurbita mixta-Vigna unguiculata, do que na monocultura de $C$. mixta.

No Brasil, são poucos os estudos sobre a ocorrência de pragas e inimigos naturais em culturas consorciadas ou em plantio direto. Bianco (1984) indicou que em sistemas de plantio direto das culturas do trigo, algodão e feijão houve redução na infestação de várias pragas. Milanez (1984) encontrou menor incidência de Anticarsia gemmatalis, Nezara viridula e Cerotoma sp. em soja consorciada com milho, enquanto Quindere \& Santos (1986) mostraram que na cultura consorciada milho-Vigna unguiculata a população de ninfas da cigarrinha, Empoasca kraemeri, foi menor, em comparação com a das ninfas observadas nas monoculturas.

Como sistemas de culturas consorciadas e de plantio direto são importantes para a agricultura, é necessário que sejam obtidas informações sobre a influência desses sistemas sobre as populações dos inimigos naturais e das pragas, para que seja melhor entendido e aproveitado o controle biológico natural. O objetivo do presente estudo foi avaliar a influência do plantio direto e da consorciação soja-milho sobre pragas e inimigos naturais.

\section{Material e Métodos}

$\mathrm{O}$ estudo foi conduzido na área experimental do Câmpus da FCAV-UNESP, em Jaboticabal, SP, durante a safra de 1997/98. A área do experimento foi de 1,6 ha, e encontrava-se em pousio desde o final da safra anterior. O trabalho foi iniciado com a aplicação do herbicida glyphosate (Randup Glifosato), na dose de 2,0 L/ha. Nas parcelas de plantio convencional foram realizadas as operações de aração e gradagem. As parcelas foram adubadas com $330 \mathrm{~kg} / \mathrm{ha}$ (fórmula 2-20-18), e não foram aplicados inseticidas para controlar pragas, durante todo o período do estudo. A semeadura foi feita em 9/12/97, usando-se a cultivar de soja BR-16 e o híbrido de milho Cargill C-701. Após a semeadura foi aplicado o herbicida metolachlor (Dual $960 \mathrm{CE}$ ), na de dose 2,0 L/ha, na área experimental.

Os tratamentos constituíram um fatorial $3 \times 2$ (monocultura de soja, monocultura de milho, consorciação soja-milho x plantio direto, plantio convencional), com blocos casualizados e quatro repetições. As parcelas com soja com $16 \times 20 \mathrm{~m}$ apresentavam 40 linhas de plantas, espaçadas de $0,5 \mathrm{~m}$. Quanto ao milho, as parcelas de 16 x $18 \mathrm{~m}$ apresentavam 20 linhas de plantas, a espaços de $0,90 \mathrm{~m}$, e as parcelas consorciadas com $16 \times 23,5 \mathrm{~m}$ eram formadas por três faixas de milho com cinco linhas de milho cada, alternadas com duas faixas de soja com dez linhas de plantas cada.

Os inimigos naturais e as pragas da soja foram amostrados no início de desenvolvimento das plantas, por procura visual, em seis metros lineares, até que as plantas atingissem a altura de $20 \mathrm{~cm}$, quando passaram a ser amostradas pelo método do pano, em três pontos/parcela, e pela rede entomológica, aplicando-se 20 redadas por parcela. Os inimigos naturais e as pragas presentes no milho foram amostrados por meio de procura visual por toda a planta e com armadilha de sucção (CDC Backpack Aspirator - Modelo 1412 movido a bateria, da Bioquip Products Inc.), aspirando-se todas as folhas e o ponteiro. Nesses dois casos, a amostragem foi feita escolhendo-se quatro pontos ao acaso dentro da parcela, amostrando-se dois metros lineares de plantas por ponto. A amostragem de insetos abrangeu todo o ciclo das culturas, em intervalos de dez dias, aproximadamente.

A ocorrência da lagarta-do-cartucho-do-milho, Spodoptera frugiperda, foi determinada contando-se o 
número de plantas danificadas, a cada dez dias, desde a germinação do milho até o estádio-3,0 (12 folhas emergidas), descrito por Fornasieri Filho (1992). A amostragem da lagarta-da-espiga-do-milho, Helicoverpa $z e a$, consistiu na retirada de dez espigas por parcela, em três épocas com intervalo de cerca de dez dias, e iniciada no momento em que se constatou a presença dessas lagartas nas espigas.

Os resultados foram submetidos à análise da variância, e as médias, comparadas pelo teste de Tukey.

\section{Resultados e Discussão}

Em relação ao milho, a interação entre o manejo do solo e culturas não foi significativa quanto ao número de insetos-pragas e de predadores anotados por meio da procura visual (Tabela 1) e amostrados com armadilha de sucção (Tabela 2). No milho, os sistemas de plantio direto e consorciação com soja pouco influíram no número de pragas; e o crisomelídeo Maecolaspis assimilis foi menos abundante no plantio direto e no milho consorciado (Tabelas 1 e 2). Estes resultados são semelhantes aos obtidos em relação a $S$. frugiperda por Castro et al. (1994), que não encontraram diferença na ocorrência desta praga em milho como monocultura e consorciado com a leguminosa Cajanus cajan (L.). Stinner et al. (1988), estudando comunidades de artrópodes em milho cultivado em áreas com 20 anos de práticas de plantio convencional, sistema de plantio reduzido e sistema de plantio direto, observaram que os danos causados por Ostrinia nubilalis e Diabrotica longicornis não foram afetados pelas práticas de manejo do solo. Em relação à soja, a interação entre o manejo do solo e culturas não foi significativa quanto ao número de insetos-pragas e predadores

Tabela 1. Número médio de insetos-pragas e de predadores anotados por meio da procura visual no milho. Jaboticabal, SP, $1997 / 98^{(1)}$

\begin{tabular}{|c|c|c|c|c|c|c|}
\hline \multirow[t]{2}{*}{ Inseto } & \multicolumn{2}{|c|}{ Manejo do solo } & \multicolumn{2}{|c|}{ Cultura } & \multirow{2}{*}{$\begin{array}{c}\text { Erro } \\
\text { padrão }\end{array}$} & \multirow{2}{*}{$\begin{array}{l}\mathrm{CV} \\
(\%)\end{array}$} \\
\hline & Direto & Convencional & Milho & Soja x milho & & \\
\hline \multicolumn{7}{|l|}{$\begin{array}{l}\text { Praga } \\
\end{array}$} \\
\hline D. speciosa & $4,1 \mathrm{a}$ & $4,8 \mathrm{a}$ & $4,5 \mathrm{a}$ & $4,5 \mathrm{a}$ & 0,77 & 30,0 \\
\hline H. zea & $5,1 \mathrm{a}$ & $5,3 a$ & $5,4 \mathrm{a}$ & $5,0 \mathrm{a}$ & 0,41 & 13,5 \\
\hline M. assimilis & $4,7 \mathrm{~b}$ & $5,9 \mathrm{a}$ & $5,9 \mathrm{a}$ & $4,7 \mathrm{~b}$ & 0,51 & 16,8 \\
\hline S. frugiperda & $4,7 \mathrm{a}$ & $5,4 \mathrm{a}$ & $5,0 \mathrm{a}$ & $5,1 \mathrm{a}$ & 1,04 & 35,8 \\
\hline \multicolumn{7}{|l|}{ Predador } \\
\hline C. sanguinea & $3,5 b$ & $4,5 \mathrm{a}$ & $3,8 \mathrm{a}$ & $4,3 \mathrm{a}$ & 0,47 & 20,1 \\
\hline Doru sp. & $35,0 \mathrm{~b}$ & $46,1 \mathrm{a}$ & $41,2 \mathrm{a}$ & $39,9 a$ & 5,20 & 22,5 \\
\hline E. conexa & $0,3 \mathrm{a}$ & $0,4 \mathrm{a}$ & $0,5 \mathrm{a}$ & $0,2 \mathrm{a}$ & 0,21 & 116,2 \\
\hline
\end{tabular}

(1) Médias seguidas da mesma letra na horizontal não diferem entre si pelo teste de Tukey a 5\% de probabilidade.

Tabela 2. Número médio de insetos-pragas e de predadores amostrados com armadilha de sucção em milho. Jaboticabal, SP, $1997 / 98^{(1)}$

\begin{tabular}{|c|c|c|c|c|c|c|}
\hline \multirow[t]{2}{*}{ Inseto } & \multicolumn{2}{|c|}{ Manejo do solo } & \multicolumn{2}{|c|}{ Cultura } & \multirow{2}{*}{$\begin{array}{c}\text { Erro } \\
\text { padrão }\end{array}$} & \multirow{2}{*}{$\begin{array}{l}\text { CV } \\
(\%)\end{array}$} \\
\hline & Direto & Convencional & Milho & $\overline{\text { Soja x milho }}$ & & \\
\hline \multicolumn{7}{|l|}{ Praga } \\
\hline L. zonatus & $2,3 \mathrm{a}$ & $1,4 \mathrm{a}$ & $1,9 \mathrm{a}$ & $1,8 \mathrm{a}$ & 1,39 & 132,7 \\
\hline \multicolumn{7}{|l|}{ Predador } \\
\hline C. sanguinea & $0,3 \mathrm{a}$ & $0,2 \mathrm{a}$ & $0,3 \mathrm{a}$ & $0,2 \mathrm{a}$ & 0,14 & 89,4 \\
\hline Doru sp. & $1,7 \mathrm{a}$ & $1,8 \mathrm{a}$ & $1,8 \mathrm{a}$ & $1,8 \mathrm{a}$ & 0,56 & 54,1 \\
\hline Orius sp. & $0,7 \mathrm{a}$ & $0,8 \mathrm{a}$ & $0,9 \mathrm{a}$ & $0,6 a$ & 0,20 & 45,8 \\
\hline Scymnus sp. & $2,6 \mathrm{a}$ & $2,8 \mathrm{a}$ & $2,7 \mathrm{a}$ & $2,7 \mathrm{a}$ & 0,84 & 54,6 \\
\hline Toxomerus sp. & $0,3 \mathrm{a}$ & $0,2 \mathrm{a}$ & $0,4 \mathrm{a}$ & $0,1 \mathrm{~b}$ & 0,12 & 95,2 \\
\hline
\end{tabular}

(1) Médias seguidas da mesma letra na horizontal não diferem entre si pelo teste de Tukey a 5\% de probabilidade. 
amostrados pelo método do pano (Tabela 3) e com rede entomológica (Tabela 4). Entre as pragas da soja, A. gemmatalis e Diabrotica gracilenta ocorreram menos no plantio direto, e observou-se maior número de Megalotomus sp. e Maecolaspis sp. na soja consorciada (Tabelas 3 e 4). Alguns autores também mostraram que o plantio direto diminuiu a abundância de espécies-pragas dessa cultura (Sloderbeck \& Yeargen, 1983).

Com relação aos predadores, foi observado menor número de Cycloneda sanguinea e Doru sp. no milho sob plantio direto do que no convencional,

Tabela 3. Número médio de insetos-pragas e de predadores amostrados pelo método do pano em soja. Jaboticabal, SP, $1997 / 98^{(1)}$

\begin{tabular}{|c|c|c|c|c|c|c|}
\hline \multirow[t]{2}{*}{ Inseto } & \multicolumn{2}{|c|}{ Manejo do solo } & \multicolumn{2}{|c|}{ Cultura } & \multirow{2}{*}{$\begin{array}{c}\text { Erro } \\
\text { padrão }\end{array}$} & \multirow{2}{*}{$\begin{array}{l}\mathrm{CV} \\
(\%)\end{array}$} \\
\hline & Direto & Convencional & Soja & Soja $\mathrm{x}$ milho & & \\
\hline \multicolumn{7}{|l|}{ Praga } \\
\hline A. gemmatalis & $3,4 \mathrm{~b}$ & $5,4 \mathrm{a}$ & $4,8 \mathrm{a}$ & $3,9 \mathrm{a}$ & 0,64 & 25,6 \\
\hline D. gracilenta & $3,9 b$ & $4,8 \mathrm{a}$ & $4,8 \mathrm{a}$ & $4,0 \mathrm{a}$ & 0,47 & 18,4 \\
\hline D. speciosa & $1,6 \mathrm{a}$ & $1,7 \mathrm{a}$ & $1,4 \mathrm{a}$ & $1,8 \mathrm{a}$ & 0,26 & 27,1 \\
\hline M. assimilis & $0,9 a$ & $1,2 \mathrm{a}$ & $1,1 \mathrm{a}$ & $1,0 \mathrm{a}$ & 0,24 & 39,2 \\
\hline Maecolaspis sp. & $0,8 \mathrm{a}$ & $0,9 \mathrm{a}$ & $0,9 \mathrm{a}$ & $0,8 \mathrm{a}$ & 0,25 & 52,4 \\
\hline Megalotomus sp. & $2,2 \mathrm{a}$ & $2,1 \mathrm{a}$ & $2,0 \mathrm{a}$ & $2,2 \mathrm{a}$ & 0,72 & 58,7 \\
\hline P. guildinii & $4,9 a$ & $3,8 \mathrm{a}$ & $3,9 \mathrm{a}$ & $4,8 \mathrm{a}$ & 0,97 & 38,4 \\
\hline \multicolumn{7}{|l|}{ Predador } \\
\hline C. sanguinea & $0,4 \mathrm{a}$ & $0,6 \mathrm{a}$ & $0,5 \mathrm{a}$ & $0,5 \mathrm{a}$ & 0,28 & 98,2 \\
\hline Geocoris sp. & $1,9 \mathrm{a}$ & $2,1 \mathrm{a}$ & $1,7 \mathrm{a}$ & $2,3 a$ & 0,38 & 32,6 \\
\hline L. concina & $1,7 \mathrm{a}$ & $1,5 \mathrm{a}$ & $1,2 \mathrm{a}$ & $2,0 \mathrm{a}$ & 0,46 & 50,3 \\
\hline Nabis sp. & $0,8 \mathrm{a}$ & $1,1 \mathrm{a}$ & $1,0 \mathrm{a}$ & $1,0 \mathrm{a}$ & 0,17 & 30,5 \\
\hline
\end{tabular}

(1) Médias seguidas da mesma letra na horizontal não diferem entre si pelo teste de Tukey a $5 \%$ de probabilidade.

Tabela 4. Número médio de insetos-pragas e de predadores amostrados com rede entomológica na soja. Jaboticabal, SP, $1997 / 98^{(1)}$.

\begin{tabular}{|c|c|c|c|c|c|c|}
\hline \multirow[t]{2}{*}{ Inseto } & \multicolumn{2}{|c|}{ Manejo do solo } & \multicolumn{2}{|c|}{ Cultura } & \multirow{2}{*}{$\begin{array}{c}\text { Erro } \\
\text { padrão }\end{array}$} & \multirow{2}{*}{$\begin{array}{l}\mathrm{CV} \\
(\%)\end{array}$} \\
\hline & Direto & Convencional & Soja & Soja $\mathrm{x}$ milho & & \\
\hline \multicolumn{7}{|l|}{ Praga } \\
\hline A. gemmatalis & $0,2 \mathrm{a}$ & $0,5 \mathrm{a}$ & $0,4 \mathrm{a}$ & $0,2 \mathrm{a}$ & 0,19 & 104,0 \\
\hline D. gracilenta & $26,1 \mathrm{a}$ & $26,5 \mathrm{a}$ & $28,2 \mathrm{a}$ & $24,5 \mathrm{a}$ & 2,85 & 19,8 \\
\hline D. speciosa & $4,2 \mathrm{a}$ & $3,7 \mathrm{a}$ & $3,3 \mathrm{a}$ & $4,5 \mathrm{a}$ & 0,64 & 28,5 \\
\hline M. assimilis & $1,7 \mathrm{a}$ & $1,9 \mathrm{a}$ & $1,5 \mathrm{a}$ & $2,1 \mathrm{a}$ & 0,32 & 30,2 \\
\hline Maecolaspis sp. & $3,8 \mathrm{a}$ & $3,9 a$ & $3,0 \mathrm{~b}$ & $4,7 \mathrm{a}$ & 0,68 & 30,8 \\
\hline Megalotomus sp. & $1,8 \mathrm{a}$ & $1,5 \mathrm{a}$ & $1,2 \mathrm{~b}$ & $2,0 \mathrm{a}$ & 0,35 & 37,0 \\
\hline Megascelis sp. & $1,5 \mathrm{a}$ & $2,8 \mathrm{a}$ & $2,3 \mathrm{a}$ & $2,0 \mathrm{a}$ & 0,94 & 76,5 \\
\hline P. guildinii & $6,2 \mathrm{a}$ & $7,5 \mathrm{a}$ & $6,7 \mathrm{a}$ & $7,0 \mathrm{a}$ & 1,26 & 31,8 \\
\hline \multicolumn{7}{|l|}{ Predador } \\
\hline C. sanguinea & $0,6 \mathrm{a}$ & $0,6 \mathrm{a}$ & $0,5 \mathrm{a}$ & $0,7 \mathrm{a}$ & 0,32 & 92,8 \\
\hline C. scutellaris & $0,2 \mathrm{a}$ & $0,3 \mathrm{a}$ & $0,1 \mathrm{a}$ & $0,3 \mathrm{a}$ & 0,20 & 154,1 \\
\hline Geocoris sp. & $3,3 \mathrm{a}$ & $3,5 \mathrm{a}$ & $2,3 b$ & $4,5 \mathrm{a}$ & 0,45 & 22,9 \\
\hline L. concina & $1,2 \mathrm{a}$ & $1,7 \mathrm{a}$ & $0,7 \mathrm{~b}$ & $2,2 \mathrm{a}$ & 0,48 & 58,3 \\
\hline Nabis sp. & $1,3 \mathrm{a}$ & $1,5 \mathrm{a}$ & $1,2 \mathrm{a}$ & $1,5 \mathrm{a}$ & 0,27 & 33,6 \\
\hline Orius sp. & $0,3 \mathrm{a}$ & $0,6 \mathrm{a}$ & $0,2 \mathrm{~b}$ & $0,6 \mathrm{a}$ & 0,21 & 81,9 \\
\hline Toxomerus & $0,2 \mathrm{a}$ & $0,3 \mathrm{a}$ & $0,3 \mathrm{a}$ & $0,2 \mathrm{a}$ & 0,16 & 119,0 \\
\hline
\end{tabular}

(1) Médias seguidas da mesma letra na horizontal não diferem entre si pelo teste de Tukey a 5\% de probabilidade. 
enquanto na soja não ocorreu diferença na abundância de predadores em nenhum dos dois tipos de plantio (Tabelas 1 a 4). Tonhasca Junior (1993) também não observou diferença na ocorrência de predadores encontrados na parte aérea da soja sob plantio direto e convencional, enquanto Buntin et al. (1995) constatou menor número de Geocoris spp. em plantio direto de soja. No consórcio do presente estudo, Geocoris sp., Lebia concina e Orius sp. foram mais abundantes na soja, ocorrendo o oposto com adultos do sirfídeo Toxomerus sp. no milho (Tabelas 1 a 4). Tonhasca Junior (1993) constatou que predadores (principalmente joaninhas e aranhas) foram mais abundantes na soja consorciada com milho do que na monocultura dessa leguminosa.
Uma das razões de a incidência de lagartas de A. gemmatalis ter sido menor na soja consorciada com milho, apesar de não-significativa, talvez tenha sido a alta taxa de predação observada, pois a densidade populacional de Orius sp., Geocoris sp. e L. concina, naquelas condições, foi significativamente mais alta (Tabela 4). Já foi demonstrado que Orius pode ter um grande impacto nas populações de lepidópteros-pragas, como, por exemplo, O. nubilalis (Jarvis \& Guthrie, 1987). A interação entre o manejo do solo e culturas não foi significativa quanto ao número de espécies e indivíduos de Hymenoptera (Tabela 5) e número de parasitóides de famílias de Hymenoptera (Tabela 6) em relação às culturas do milho e da soja. Entre parasitóides da

Tabela 5. Número médio de espécies e indivíduos de Hymenoptera amostrados em milho (armadilha de sucção) e soja (rede entomológica). Jaboticabal, SP, 1997/98(1).

\begin{tabular}{lccccc}
\hline Família & \multicolumn{2}{c}{ Milho } & & \multicolumn{2}{c}{ Soja } \\
\cline { 2 - 3 } \cline { 5 - 6 } & Espécie & Indivíduo & & Espécie & Indivíduo \\
\hline Braconidade & 3 & 6 & 6 & 240 \\
Encyrtidae & 1 & 2 & & 1 & 280 \\
Eucoilidae & 1 & 7 & & 2 & 54 \\
Eulophidae & 5 & 185 & 6 & 100 \\
Ichneumonidae & 1 & 2 & 2 & 6 \\
Mymaridae & 0 & 0 & 1 & 6 \\
Platygasteridae & 0 & 53 & & 1 & 3 \\
Pteromalidae & 1 & 2 & & 10 \\
Scelionidae & 1 & 0 & & 1 & 285 \\
Trichogrammatidae & 0 & 257 & 29 & 2360 \\
\hline Total & 13 & & & & 2344 \\
\hline
\end{tabular}

Tabela 6. Número médio de parasitóides de famílias de Hymenoptera capturados em milho (armadilha de sucção) e soja (rede entomológica). Jaboticabal, SP, 1997/98(1).

\begin{tabular}{|c|c|c|c|c|c|c|}
\hline \multirow[t]{2}{*}{ Família } & \multicolumn{2}{|c|}{ Manejo do solo } & \multicolumn{2}{|c|}{ Cultura } & \multirow{2}{*}{$\begin{array}{c}\text { Erro } \\
\text { padrão }\end{array}$} & \multirow{2}{*}{$\begin{array}{l}\mathrm{CV} \\
(\%)\end{array}$} \\
\hline & Direto & Convencional & Monocultura & Soja $\mathrm{x}$ milho & & \\
\hline \multicolumn{7}{|l|}{ Milho } \\
\hline Eulophidae & $10,5 \mathrm{a}$ & $11,4 \mathrm{a}$ & $11,1 \mathrm{a}$ & $10,8 \mathrm{a}$ & 1,54 & 28,3 \\
\hline Pteromalidae & $3,5 \mathrm{a}$ & $2,5 \mathrm{a}$ & $3,1 \mathrm{a}$ & $2,9 \mathrm{a}$ & 0,64 & 43,0 \\
\hline Eucoilidae & $0,2 \mathrm{a}$ & $0,1 \mathrm{a}$ & $0,3 \mathrm{a}$ & $0,1 \mathrm{a}$ & 0,10 & 133,6 \\
\hline \multicolumn{7}{|l|}{ Soja } \\
\hline Braconidae & $2,9 a$ & $3,1 \mathrm{a}$ & $1,2 \mathrm{~b}$ & $4,8 \mathrm{a}$ & 0,95 & 64,5 \\
\hline Encyrtidae & $3,2 \mathrm{a}$ & $3,8 \mathrm{a}$ & $3,7 \mathrm{a}$ & $3,3 \mathrm{a}$ & 0,70 & 40,3 \\
\hline Eucoilidae & $1,0 \mathrm{a}$ & $1,1 \mathrm{a}$ & $1,1 \mathrm{a}$ & $1,0 \mathrm{a}$ & 0,29 & 54,8 \\
\hline Eulophidae & $1,8 \mathrm{a}$ & $1,2 \mathrm{~b}$ & $1,5 \mathrm{a}$ & $1,5 \mathrm{a}$ & 0,22 & 29,9 \\
\hline Scelionidae & $3,0 \mathrm{a}$ & $3,9 a$ & $2,0 \mathrm{a}$ & $5,0 \mathrm{~b}$ & 0,58 & 33,6 \\
\hline Trichogrammatidae & $15,0 \mathrm{~b}$ & $27,8 \mathrm{a}$ & $17,6 \mathrm{a}$ & $25,2 \mathrm{a}$ & 3,68 & 34,4 \\
\hline
\end{tabular}

(1) Médias seguidas da mesma letra na horizontal não diferem entre si pelo teste de Tukey a 5\% de probabilidade. 
ordem Hymenoptera, foram capturadas, no milho, sete famílias, das quais Eulophidae e Pteromalidae representaram $92,6 \%$ do total, enquanto na soja, das dez famílias encontradas, Trichogrammatidae, Scelionidae, Encyrtidae, Braconidae, Eulophidae e Eucoilidae abrangeram 98,9\% do total (Tabela 5). No milho, foi observada a ocorrência de Conura sp. (Chalcididae), que é indicada como hiperparasitóide de Braconidae. Entre as famílias que foram encontradas mais consistentemente no milho, não houve diferença na sua ocorrência, quer seja com relação ao plantio direto quer com relação à consorciação (Tabela 6). Por outro lado, foi observado maior número de parasitóides das famílias Eulophidae e Trichogrammatidae, na soja cultivada, respectivamente, sob plantio direto e convencional, enquanto espécies das famílias Braconidae e Scelionidae foram mais abundantes na soja consorciada (Tabela 6). Vários autores também observaram maior abundância de parasitóides em culturas consorciadas, como milho e batata-doce (Nafus \& Schreiner, 1986), milho-Cucurbita mixta-Vigna unguiculata (Letourneau, 1987) e milho-algodão (Shalaby et al., 1988).

A presença do milho pode ter sido um dos fatores que expliquem a maior abundância de vários inimigos naturais observados na soja consorciada (Tabelas 4 e 6). Deste modo, o milho pode ter afetado a turbulência do vento nas faixas de soja; quebra-ventos são conhecidos por aumentarem a concentração de inimigos naturais em soja (Mayse \& Price, 1978). O microclima nas faixas de soja pode ter sido alterado, pelo fato de ocorrer mais sombra, umidade mais alta, e temperaturas mais baixas, fatores que podem aumentar o número de inimigos naturais em culturas diversificadas que incluam o milho (Letourneau, 1987). Além disso, já foi observado que o milho forneceu alimento para predadores. Coleomegilla maculata e outras joaninhas já foram observadas se alimentando de pólen de milho depositado em folhas de soja (Risch et al., 1982).

Entre os métodos utilizados na avaliação da ocorrência de insetos, a rede entomológica proporcionou a captura de maior diversidade e número destes organismos, com menor gasto de tempo. Deve ser destacado que a armadilha de sucção foi imprescindível para a captura de parasitóides presentes nas plantas de milho.

\section{Conclusões}

1. A ocorrência de pragas é menor na soja e milho no sistema plantio direto.

2. O consórcio soja-milho proporciona maior ocorrência de inimigos naturais na soja.

\section{Agradecimentos}

À FAPESP, pelo apoio financeiro para a realização deste trabalho; ao CNPq, pela bolsa Produtividade em Pesquisa fornecida ao primeiro autor; ao Dr. Nelson W. Perioto, do Instituto Biológico, pela identificação dos parasitóides; ao Dr. Carlos Campaner, do Museu de Zoologia-USP, pela identificação de crisomelídeos e carabídeos.

\section{Referências}

ANDOW, D. A. Vegetational diversity and arthropod population response. Annual Review of Entomology, Palo Alto, v. 36, p. 561-586, 1991.

BECHINSKI, E. J.; PEDIGO, L. P. Ecology of predaceous arthropods in Iowa soybean agroecosystems. Environmental Entomology, Lanham, v. 10, p. 771-778, 1981.

BEST, L. R.; BEEGLE, C. C. Food preferences of five species of carabids commonly found in Iowa cornfields. Environmental Entomology, Lanham, v. 6, p. 9-12, 1977.

BIANCO, R. Ocorrência de pragas no plantio direto $x$ convencional. In: FANCELLI, A. L.; TORRADO, P. V.; MACHADO, J. (Coord.). Atualização em plantio direto. Campinas : Fundação Cargill, 1984. p. 183-194.

BRUST, G. E.; STINNER, B. R.; McCARTNEY, D. A. Predation by soil inhabiting arthropods in intercropped and monoculture agroecosystems. Agriculture, Ecosystems and Environment, Amsterdam, v. 18, p. 145-154, 1986.

BUNTIN, G. D.; HARGROVE, W. L.; McCRAKEN, D. V. Populations of foliage-inhabiting arthropods on soybean with reduced tillage and herbicide use. Agronomy Journal, Madison, v. 87, p. 789-794, 1995.

CASTRO, M. T.; PITRE, H. N.; MECKENSTOCK, D. H. Fall armyworm and neotropical cornstalk borer on sorghum and maize intercropped with legumes in Honduras. Turrialba, San Jose, v. 44, p. 77-86, 1994. 
EMDEN, H. F. van; WILLIAMS, G. F. Insect stability and diversity in agro-ecosystems. Annual Review of Entomology, Palo Alto, v. 19, p. 455-474, 1974.

FORNASIERI FILHO, D. A. Cultura do milho. Jaboticabal : FUNEP, 1992. 273 p.

FULLER, B. W. Predation by Calleida decora (F.) (Coleoptera: Carabidae) on velvetbean caterpillar (Lepidoptera: Noctuidae) in soybean. Journal of Economic Entomology, Lanham, v. 81, p. 127-129, 1988.

HOUSE, G. J.; STINNER, B. R. Arthropods in no-tillage soybean agroecosystems: community composition and ecosystem interactions. Environmental Entomology, Lanham, v. 7, p. 23-28, 1983.

HSIN, C. Y.; SELLERS, L. G.; DAHM, P. A. Seasonal activity of carabids and the toxicity of carbofuran and terbofos to Pterostichus chalcites. Environmental Entomology, Lanham, v. 8, p. 154-159, 1979.

JARVIS, J. L.; GUTHRIE, W. D. Ecological studies of the European corn borer (Lepidoptera: Pyralidae) in Boone County, Iowa. Environmental Entomology, Lanham, v. 16 , p. 50-58, 1987.

LETOURNEAU, D. K. The enemies hypothesis: tritrophic interactions and vegetational diversity in tropical agroecosystems. Ecology, Washington, v. 68, p. 1616-1622, 1987.

MAYSE, M. A.; PRICE, P. W. Seasonal development of soybean arthropod communities in East Central Illinois. Agro-Ecosystems, Amsterdam, v. 4, p. 387-405, 1978.

MILANEZ, J. M. Ocorrência de insetos em culturas consorciadas e monoculturas. In: ENCONTRO NACIONAL DE FITOSSANITARISTAS, 3., 1984, Florianópolis. Anais... Florianópolis : Ministério da Agricultura, 1984. p. 103-115.

NAFUS, D.; SCHREINER, I. Intercropping maize and sweet potatoes: effects on parasitization of Ostrinia furnicalis eggs by Tricogramma chilonis. Agriculture,
Ecosystems and Environment, Amsterdam, v. 15 p. 189-200, 1986.

QUINDERE, M. A. W.; SANTOS, J. H. R. dos. Efeito de época relativa de plantio no consórcio milho $\mathrm{x}$ caupi sobre a presença de insetos úteis e o manejo econômico das pragas. Pesquisa Agropecuária Brasileira, Brasília, v. 21 , n. 4, p. 355-368, abr. 1986.

RISCH, S. J.; ANDOW, D. A.; ALTIERI, M. A. Agroecosystem diversity and pest control: data, tentative conclusions and, new research directions. Environmental Entomology, Lanham, v. 12, p. 625-629, 1983.

RISCH, S. J.; WRUBEL, R.; ANDOW, D. Foraging by a predaceous beetle, Coleomegilla maculata (Coleoptera: Coccinellidae), in a polyculture: effects of plant density and diversity. Environmental Entomology, Lanham, v. 11, p. 949-950, 1982.

SHALABY, F. F; ABDEL-GAWAAD, A. A.; IBRAHIM, A. A.; KARES, E. A. Effect of intercropping maize in cotton fields on the population of the cotton leaf-worm parasites, their efficiency and the economic income. Entomological Society of Egypt Bulletin, Economic Series, Cairo, v. 15, p. 33-46, 1988.

SLODERBECK, P. E.; YEARGEN, K. V. Green cloverworm (Lepidoptera: Noctuidae) populations in conventional and double-cropped, no-till soybeans. Journal of Economic Entomology, Lanham, v. 76, p. $785-791,1983$.

STINNER, B. R.; McCARTNEY, D. A.; DOREN JUNIOR, D. M. van. Soil and foliage arthropod communities in conventional, reduced and no-tillage corn (maize, Zea mays L.) systems: a comparison after 20 years of continuous cropping. Soil and Tillage Research, Amsterdam, v. 11, p. 147-158, 1988.

TONHASCA JUNIOR, A. Effects of agroecosystem diversification on natural enemies of soybean herbivores. Entomologia Experimentalis et Applicata, Dordrecht, v. 69 , p. $83-90,1993$. 